\title{
André Sauzeau, Pierre Sauzeau, La quatrième fonction. Altérité et marginalité dans l'idéologie des Indo-Européens
}

Paris, Les Belles Lettres, coll. «Vérité des mythes », 2012, 410 p.

Jean-Bruno Renard

\section{OpenEdition \\ Journals}

Édition électronique

URL : http://journals.openedition.org/assr/27457

DOI : $10.4000 /$ assr. 27457

ISSN : $1777-5825$

Éditeur

Éditions de l'EHESS

Édition imprimée

Date de publication : 1 octobre 2015

Pagination : 368

ISBN : 978-2-7132-2515-4

ISSN : 0335-5985

\section{Référence électronique}

Jean-Bruno Renard, «André Sauzeau, Pierre Sauzeau, La quatrième fonction. Altérité et marginalité dans l'idéologie des Indo-Européens ", Archives de sciences sociales des religions [En ligne], 172 I octobre-décembre, mis en ligne le 21 juin 2016, consulté le 24 septembre 2020. URL : http:// journals.openedition.org/assr/27457 ; DOI : https://doi.org/10.4000/assr.27457 


\section{André Sauzeau, Pierre Sauzeau, La quatrième fonction. Altérité et marginalité dans l'idéologie des Indo-Européens}

Paris, Les Belles Lettres, coll. « Vérité des mythes », 2012, 410 p.

Jean-Bruno Renard

\section{RÉFÉRENCE}

André Sauzeau, Pierre Sauzeau, La quatrième fonction. Altérité et marginalité dans l'idéologie des Indo-Européens, Paris, Les Belles Lettres, coll. « Vérité des mythes », 2012, 410 p. 
1 Pour rendre compte de cet ouvrage, il faut d'abord rappeler brièvement la découverte majeure de Georges Dumézil (1898-1986). Pour le célèbre mythologue, les anciens peuples indo-européens partagent une même vision du monde qui structure la religion, les mythes et la représentation de la société selon trois fonctions: la fonction magico-religieuse et juridique (F1), la fonction guerrière (F2) et la fonction productrice (F3). Dumézil a mis au jour cette «idéologie trifonctionnelle» dans de multiples cultures: indienne, grecque, romaine, germanique, celtique et scandinave. Les dieux des polythéismes se répartissent dans les trois fonctions et certaines sociétés s'organisent en groupes

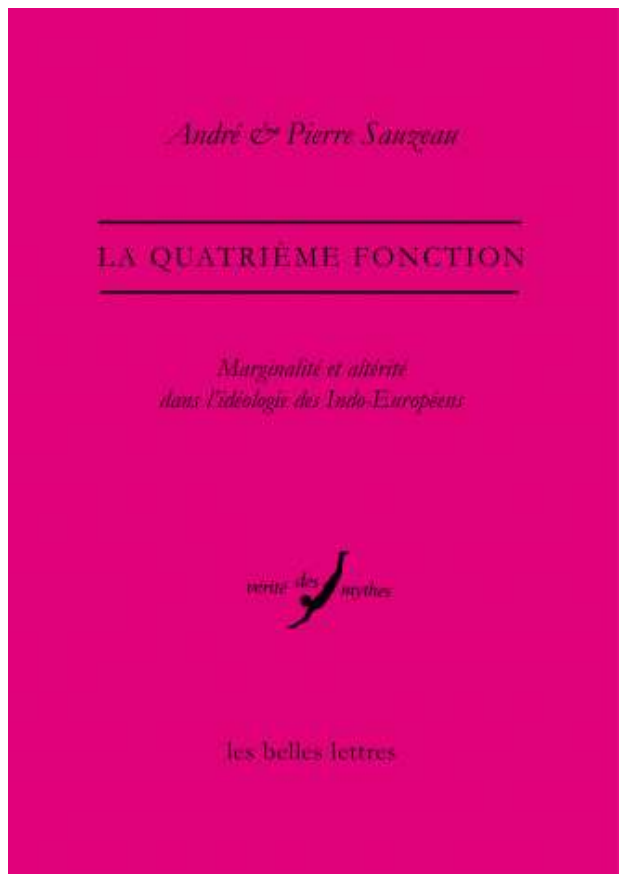
fonctionnels comme les trois grandes castes indiennes (Brahmanes, Kshatriya, Vaishya) ou, jusqu'au XVIII ${ }^{\mathrm{e}}$ siècle, les trois Ordres de l'Ancien Régime en France (Clergé, Noblesse, Tiers-État).

2 Pierre et André Sauzeau énoncent clairement leur objectif : « Notre ouvrage n'entend pas remettre en cause l'œuvre de Dumézil, mais la poursuivre. Il s'agit d'élargir le modèle dumézilien, et précisément de proposer une structure quadrifonctionnelle. Cette quatrième fonction, en charge du non-Ordre, de l'ambiguïté, de la transformation, de la marginalité, de l'altérité, complète de façon économique le modèle trifonctionnel » (p.14). Quelques précurseurs avaient déjà émis cette idée d'une quatrième fonction. Alwyn et Brinley Rees, spécialistes du domaine celtique (Celtic Heritage, 1961), ont décelé une quatrième fonction dans la quatrième caste de l'Inde (les Serviteurs ou Shudra) et ont vu dans le symbolisme associé à la quatrième province de l'Irlande (le Munster), qui contrairement aux trois autres ne relève d'aucune fonction dumézilienne, l'expression des thèmes de la mort et de l'étrangeté. Plus tard, l'anthropologue de l'Inde Nick Allen a commencé à théoriser l'hypothèse quadrifonctionnelle (« The Ideology of the IndoEuropeans: Dumézil's Theory and the Idea of a Fourth Function », International Journal of Moral \& Social Studies, 1987, 2, p. 23-39).

On pouvait s'attendre à ce passage de trois à quatre puisqu'en bonne logique structurale un ensemble A (ici les trois fonctions d'Ordre) ne prend son sens que par opposition à non-A (ici le Désordre). Par ailleurs, bien qu'il ne soit pas cité par les auteurs, Carl Gustav Jung et son archétype de la quaternité ne sont pas très éloignés de la thèse de la $4^{\mathrm{e}}$ fonction : Charles Baudouin (L'CEuvre de Jung, 1963, p. 192) mentionne en effet « le motif, recueilli par Jung à de forts nombreux exemplaires, des quatre objets ou des quatre personnages, dont trois forment bloc tandis que le quatrième fait bande à part; ou encore trois sont présents et le quatrième absent: ce qui marque bien une incomplétude aspirant à se dépasser ».

4 Les auteurs montrent qu'un grand nombre des difficultés auxquelles se sont heurtés Georges Dumézil et ses successeurs peuvent être résolues par le modèle 
quadrifonctionnel, sans qu'il soit nécessaire de minimiser ou d'écarter des données comme atypiques, d'arguer de spécificités locales, ou encore d'expliquer une quaternité par le dédoublement d'une des trois fonctions canoniques ou par l'inclusion des trois fonctions dans un schéma quaternaire (4 points cardinaux, 4 saisons ou 4 éléments). Les auteurs, tout en acceptant la thèse selon laquelle les anciens peuples indo-européens structurent le monde ordonné suivant un schéma trifonctionnel, ajoutent deux hypothèses :

- Ces peuples connaissent l'existence d'un non-Ordre.

- Pour couvrir la totalité du monde, il faut inclure un $4^{e}$ élément comme complémentaire, extérieur, hostile ou étranger.

7 Les 21 chapitres qui composent cet ouvrage de 410 pages déroulent de multiples études de cas pour démontrer la validité scientifique et heuristique de l'élargissement du modèle. L'imposante bibliographie de près de 580 références témoigne du sérieux et de l'érudition des auteurs. À titre d'exemple, on présentera succinctement quelques démonstrations particulièrement convaincantes.

Dans la Grèce archaïque, les couleurs fondamentales sont au nombre de trois : le blanc, le rouge et le noir. En conformité avec la grille d'analyse dumézilienne, le blanc céleste et lumineux est associé à la fonction religieuse (F1), le rouge sanguin est associé à la fonction guerrière (F2) et le noir terreux correspond à la fonction productive (F3). Mais il existe aussi une couleur trouble, le « chlore ", qui oscille entre le blanc livide, le bleu pâle, le jaunâtre et le verdâtre. Symboliquement associé à des éléments négatifs comme la bile, la maladie, la peur et la mort, le chlore est la couleur de la quatrième fonction (F4). Imprégné de culture grecque, l'évangéliste Jean reprendra ces couleurs pour décrire les chevaux des quatre cavaliers de l'Apocalypse qui viennent semer la destruction dans le monde : le cheval blanc est celui du Juge royal (F1), le cheval rouge est monté par un cavalier armé d'une épée (F2), le cheval noir porte le cavalier muni d'une balance pour peser le pain et l'huile (F3) et le cheval verdâtre est chevauché par un squelette, symbolisant la mort ou la peste (F4).

9 Les rites initiatiques peuvent être interprétés comme la sortie, puis la réintégration de l'initié dans le système trifonctionnel, après un passage par la $4^{\mathrm{e}}$ fonction, c'est-à-dire "une traversée dans l'Ailleurs non ordonné » (p.133). Ainsi, dans un récit gallois médiéval, le héros reçoit un nom (F1), des armes (F2), une femme (F3) et doit subir une mort initiatique (F4) pour ensuite accéder au trône, comme synthèse royale des trois premières fonctions.

10 L'épisode célèbre du Jugement de Pâris correspond bien à l'analyse dumézilienne classique : les trois femmes parmi lesquelles le jeune homme doit choisir la plus belle sont la déesse Héra, sœur et épouse de Zeus (F1), Athéna, déesse de la guerre (F2), et Aphrodite, déesse de l'amour et de la fécondité (F3). Selon les frères Sauzeau, qui reprennent une intuition de Nick Allen, la quatrième fonction est incarnée par Pâris lui-même : associé à Hermès, le dieu des passages, lié au monde animal (il est berger) et à la transe (il danse), Pâris figure F4, le non-Ordre extérieur à l'Ordre.

11 Les temps sociaux sont également distribués selon les quatre fonctions : le temps de la prière (F1), le temps de la guerre (F2), le temps du travail (F3) mais aussi le temps des fêtes d'inversion - Lupercales, Saturnales - (F4) qui symbolisent le non-Ordre nécessaire pour compléter le monde. « Les fêtes carnavalesques marquent l'entrée dans l'altérité où se ressource le monde »(p. 193). 

ou telle des trois fonctions duméziliennes, il en est qui demeurent inclassables. Ces divinités sont celles des passages, des carrefours, de la ruse, des métamorphoses, de l'animalité sauvage et de la mort. Ces dieux de la quatrième fonction sont Rudra dans l'Inde védique, Shiva dans l'Inde hindouiste, Chronos, Hermès, Hécate et Pan en Grèce, Saturne, Mercure, Janus et Faunus à Rome, Ullr dans le panthéon nordique et Loki pour les Scandinaves. Une figure mythique emblématique de la quatrième fonction est le Trickster, le Fripon divin, dieu, demi-dieu ou héros qui trompe les dieux par la ruse et transgresse l'ordre du monde, pour établir un nouvel ordre. Les personnages mythologiques présentent souvent des aspects relevant des fonctions d'Ordre et de la fonction de Désordre: ainsi Hercule (F2) combat les monstres infernaux (F4) pour s'approprier leur force; la Terre est le séjour des morts (F4) mais aussi le réservoir de la fertilité (F3).

Citons pour finir la belle analyse (chap. XVI) du chant V de l'Énéide où Virgile raconte une course de quatre navires. La grille de lecture quadrifonctionnelle se révèle très pertinente. Le symbolisme des navires reflète parfaitement les quatre fonctions. La Scylla gagne la course grâce à la piété de ses navigateurs, qui se voient remettre des trépieds sacrés en récompense (F1). La Pristis arrive en deuxième place, grâce à sa rapidité et à un « équipage ardent » qui reçoit en récompense des armes et des étoffes pourpres (F2). Le troisième navire est la Chimère, un bateau énorme où se pressent de nombreux marins, qui recevront à l'arrivée des pièces d'or et d'argent (F3). Quant au Centaure, après avoir heurté des récifs, il arrive bon dernier et reçoit une esclave comme «prix de consolation» (F4). Comme l'écrivent Pierre et André Sauzeau, «les régates en l'honneur d'Anchise constituent un bon exemple du rôle de matrice imaginaire que peut jouer la structure quadrifonctionnelle pour la genèse d'un mythe ici sans doute d'un "mythe littéraire" » (p. 274).

La finesse d'analyse des auteurs s'applique aussi à des données iconographiques, comme le montre le chapitre XX où sont étudiés le bol d'or d'Hasanlu, pièce iranienne $\mathrm{du} \mathrm{Ix}^{\mathrm{e}}$ siècle avant J.-C., ainsi que le pilier du Zbruch, monolithe sculpté slave du $\mathrm{IX}^{\mathrm{e}}$ siècle après J.-C., pour lequel Georges Dumézil avait proposé une interprétation.

Infatigables chercheurs, les auteurs annoncent des ouvrages en préparation, dans la continuité de celui-ci, notamment un prochain volume qui éclairera par la quatrième fonction les épopées indiennes et grecques ainsi que les mythes eschatologiques.

Les travaux de Pierre et André Sauzeau présentent un double intérêt. Non seulement ils offrent une nouvelle clé de lecture pour les mythes et les rites des peuples indoeuropéens, mais encore, au-delà du modèle tri ou quadrifonctionnel, ils ouvrent à la problématique universelle de la marginalité et de l'altérité, cette " part maudite » que Georges Bataille voulait étudier dans son projet philosophique et anthropologique d'une science nouvelle, l'« hétérologie ». 